\title{
A simple, cost effective method for isolation and transportation of Leishmania parasites
}

\author{
R L Ihalamulla', U S Rajapaksa ${ }^{1}$, H V Y D Siriwardena ${ }^{1}$, M Chance $^{2}$, N D Karunaweera ${ }^{1}$ \\ (Index words: cost effective, transportation, Leishmania, Sri Lanka)
}

\begin{abstract}
Isolation and propagation of Leishmania parasites using conventional culture methods are difficult, especially under field conditions. Transportation of live parasites requires the maintenance of low temperatures, which increases the cost considerably. The present study demonstrates that monophasic micro capillary culture is a simpler, more economical and better alternative to using conventional Evan's modified Tobie's medium to isolate and transport the Leishmania parasite over long distances without the use of temperature control methods.
\end{abstract}

\section{Introduction}

Isolation, growth and propagation of Leishmania parasites from skin lesions of patients suspected of having cutaneous leishmaniasis need technical expertise and sterile conditions. These requirements are difficult to meet especially under field conditions. Use of conventional, biphasic media, such as, the Novy, McNeal and Nicolle (NNN) and Evan's modified Tobie's for isolation in a field facility has many disadvantages. These include, the larger size of vials or glass test tubes in which the biphasic medium is layered, temperature maintenance at about $4^{\circ} \mathrm{C}$ and the need to hold tubes in the upright position. Test tube racks and insulated containers packed with ice which are large enough to accompany the racks are, therefore, necessary. This makes the field kits bulky, resulting in a high cost for transportation.

Monophasic microculture maintained in capillary tubes [1] on the other hand, could be carried at minimal transport cost and with a lower risk of contamination of the cultures during inoculation [2]. The standard method used for transportation of isolated parasites to overseas laboratories is by air in freezing-vials $(1.0 \mathrm{ml})$, which in turn are kept in an insulated container, packed in dry-ice, which adds to the weight and cost of transportation. The present study demonstrates successful long-distance transportation of live parasites inoculated into microcultures without temperature control measures, which makes this an effective and inexpensive method.

\section{Materials and methods}

Sterile saline aspirates of about $150 \mu$ l each were collected into eppendorf tubes under field conditions, near a lighted candle, from 28 patients who were suspected of having cutaneous leishmaniasis, after obtaining informed consent. Ethical clearance for sample collection was obtained from the Ethical Review Committee of the Faculty of Medicine, University of Colombo. Microcultures were made from aspirates collected from these patients as described previously [2]. Cultures were incubated at ambient temperature $\left(27-33^{\circ} \mathrm{C}\right)$. These were examined under an inverted microscope for promastigotes from the second day of incubation. Visualization of promastigotes was considered as evidence of culture positivity. Seven such promastigote-positive cultures in capillary tubes were prepared for long distance transportation placing them between two layers of hard cardboard with a groove to accommodate them and stabilized using gum tape. Three such cultures were sent to Laboratoire de Parasitologie in Montpellier, France. The balance four capillary cultures packed in the same manner, were sent to the Liverpool School of Tropical Medicine, United Kingdom. Both packages were transported at ambient temperature.

\section{Results and discussion}

All 28 cultures that were inoculated were found to be promastigote-positive with no bacterial contamination, further confirming the usefulness of microcultures for parasite isolation under field conditions [2]. Parasites remained positive in cultures maintained at room temperature for 30 to 40 days after inoculation. The three cultures sent to France reached the destination after 14 days of posting and were found to be viable on receipt. The promastigotes in these cultures were subsequently mass cultured successfully and were identified as Leishmania donovani using molecular methods (J. P. Dedet, personal communication). The four cultures sent to Liverpool were found to be viable after 10 days of transportation. All except one of these were mass cultured successfully. 
This study demonstrates the ability of Leishmania promastigotes to grow and multiply in microcultures at ambient temperature. This feature of the Sri Lankan Leishmania parasite, to grow in the microcapillary environment at ambient temperature for a considerable length of time (up to 40 days in this study) is a great advantage for long distance transportation. This reduces cost since excessive packing with ice and insulation are not required, and conventional mailing techniques could be adopted.

\section{Conclusion}

The microcapillary culture method seems a simple, safe and economical method for field isolation, and long distance transportation of Leishmania parasites.

\section{Acknowledgements}

The authors thank Professor J. P. Dedet of Laboratoire de Parasitologie, Montpellier, France for assistance in mass culture techniques and identification of the Leishmania isolates sent in capillary cultures. They also wish to thank Mr. K. H.Weerasena and Mr. Saman Jayasinghe of the Faculty of Medicine, Colombo, for assistance in obtaining samples from patients. This study was supported financially by the National Science Foundation (grant RG/ SIDA/2003/M/01) and a grant received by YS from the University Grants Commission.

\section{References}

1. Allahverdiyev A, Uzum S, Bagirova M, Menisoglu HR. A sensitive new microculture method for diagnosis of cutaneous leishmaniasis. American Journal of Tropical Medicine and Hygiene 2004; 70: 294-7.

2. Ihalamulla RL, Rajapaksa US, Karunaweera ND. Microculture for the isolation of Leishmania parasites from cutaneous lesions - Sri Lankan experience. Annals of Tropical Medicine and Parasitology 2005; 99: 571-5. 